\title{
Invariant grating pseudoimaging using polychromatic light and a finite extension source
}

\author{
Luis Miguel Sanchez-Brea, ${ }^{1, *}$ Jose Saez-Landete, ${ }^{2}$ Jose Alonso, ${ }^{1}$ and \\ Eusebio Bernabeu ${ }^{1}$ \\ 'Department of Optics, Applied Optics Complutense Group, Facultad de Ciencias Físicas, \\ Universidad Complutense de Madrid Ciudad Universitaria s.n., Madrid 28040, Spain \\ ${ }^{2}$ Departamento de Teoría de la Señal y Comunicaciones, Escuela Politécnica Superior, Universidad de Alcalá, \\ Campus Universitario, Alcalá de Henares, Madrid 28871, Spain \\ *Corresponding author: sanchezbrea@fis.ucm.es
}

Received 16 October 2007; revised 30 January 2008; accepted 6 February 2008; posted 7 February 2008 (Doc. ID 88667); published 27 March 2008

\begin{abstract}
The Talbot effect is a well studied phenomenon by which grating pseudoimages appear at certain periodic distances when monochromatic light is used. Recently, numerical simulations have shown a new phenomenon; when a polychromatic light beam is used in a double grating system, the intensity of the pseudoimages presents a transverse-profile that remains unaffected over a wide range of propagation distances. This effect can be used to increase the tolerances of gratings based optical devices, such as displacement measurement systems, interferometers, and spectrometers. The pseudoimages formation with a polychromatic and finite extension light source is analytically and experimentally demonstrated. Relatively simple analytical expressions for the intensity and the contrast allow us to predict when pseudoimages present a constant contrast and when they disappear. Furthermore, we experimentally obtain the pseudoimages using the proposed configuration, corroborating the theoretical predictions. (C) 2008 Optical Society of America
\end{abstract}

OCIS codes: $\quad 050.0050,050.2770$.

\section{Introduction}

Double grating systems are used in many applications such as, for example, in displacement measurement systems, interferometers, and spectrometers [1-4]. From the first studies based on diffraction theory [5], a model for pseudoimage formation based on Fresnel propagation of light has been progressively improved [6,7], introducing total incoherence [8], partially coherent light [ $\underline{9}-\underline{13}$ ] and finite spectral width [14].

At the moment, most applications of double grating systems use pseudoimage fringes, which are generated at finite distances from the second grating. Then, no lenses are required, and the devices are more compact and robust. Usually, in these config-

0003-6935/08/101470-08\$15.00/0

(C) 2008 Optical Society of America urations, the fringes contrast strongly depends on the distance between gratings, since a similar condition to the Talbot effect must be fulfilled. This condition is needed to obtain significant contrast pseudoimages [8]. On the other hand, new technologies are demanding systems with higher resolutions, therefore, the use of diffraction gratings with lower and lower grating periods. The condition of pseudoimage formation depends on the gratings periods, as a consequence, tiny perturbations of the grating gap may vanish the contrast, even a contrast inversion can happen. In grating measurement systems, a contrast inversion produces an error in the measure.

For the Talbot effect, a solution to this problem has been obtained using polychromatic light $[15,16]$. Double grating systems with polychromatic light have also been numerically analyzed, showing that some pseudoimages maintain a high contrast while other pseudoimages disappear [14]. The appearance, 
disappearance, and stability of the pseudoimages can be seen in a contrast representation with respect to $z_{1}$ and $z_{2}, z_{1}$ being the distance between gratings and $z_{2}$ the distance between the second grating and the observation plane. In current applications, LEDs are normally used as light sources with sizes ranging from 50 to $1000 \mu \mathrm{m}, 300 \mu \mathrm{m}$ being the most common size. Therefore, the finite size of the light source has to be considered in the analysis. For standard grating periods and source sizes, pseudoimages only present a significant contrast at narrow bands in the $\left(z_{1}, z_{2}\right)$ plane (pseudoimage isolation) [14]. This corresponds to regime 3 of the said papers.

In the present paper, we analytically and experimentally investigate why some pseudoimages in this third regime maintain a high contrast over a wide propagation distance when polychromatic light is used, while other pseudoimages disappear. For this, we analyze the intensity distribution of a double grating system with polychromatic light and a finite size. The general expression is too complicated to analyze the physical behavior of double grating systems, except for very simple cases $[17,18]$. In this paper, without loss of generality, we simplify the general expression in order to obtain the contrast of the pseudoimages in terms of the distance between the second grating and the observation plane. Furthermore, experimental results demonstrate the validity of the analytical approach and the final results. To our knowledge, in the literature there is no experimental verification of constant contrast pseudoimages when polychromatic light is used. This phenomenon can be applied, for example, for improving the mechanical tolerances of grating based displacement measurement systems. Several commercial devices use the generalized grating imaging configuration. One of the gratings is in the scanning head, along with the light source and the photodetectors, and the other diffraction grating is in a mobile stage. For a standard diffraction grating period of $10 \mu \mathrm{m}$, the distance between the Talbot planes is $\sim 200 \mu \mathrm{m}$. When monochromatic light is used, then the distance between the gratings should be maintained with a mechanical tolerance of $\sim 20-40 \mu \mathrm{m}$, which it is too tight to be fulfilled.

\section{Grating Pseudoimaging with Polychromatic Light}

Let us consider a double grating system as depicted in Fig. 1. We will assume a finite size source made up of point emitters, which incoherently generate divergent spherical waves. The lateral size of the light source is $S$. Through this model, the spatial coherence of the field can be selected by changing the size and location of the source. The distance between the light source and the first grating is $z_{0}$, and the distance between both gratings is $z_{1}$. The periods of the gratings $G_{1}$ and $G_{2}$ are $p_{1}$ and $p_{2}$, respectively. These periods can be equal or different, and they are assumed much larger than the wavelength, accordingly, the scalar diffraction approach can be used. The gratings are amplitude or phase gratings

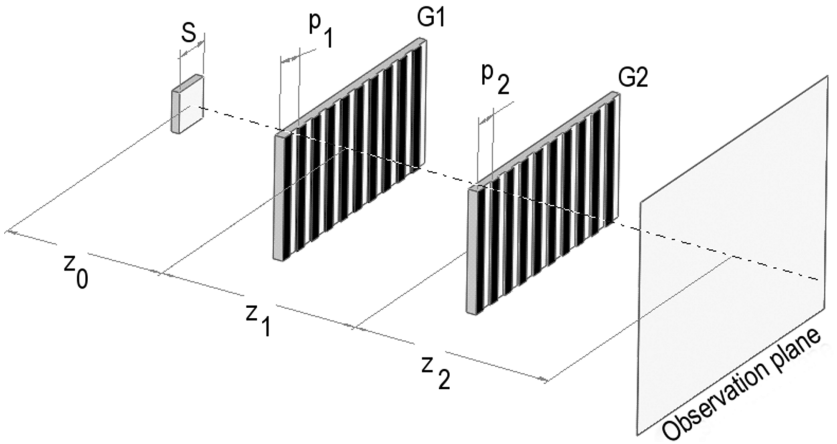

Fig. 1. Double grating configuration. The light source presents a finite size $S$, and it emits a polychromatic light distribution.

with an arbitrary profile, and they are characterized by means of the complex transmittance $t_{1}(x)=$ $\sum_{n} c_{1, n} \exp \left(i n q_{1} x\right), \quad t_{2}(x)=\sum_{m} c_{2, m} \exp \left(i m q_{2} x\right)$, where $q_{1}=2 \pi / p_{1}$ and $q_{2}=2 \pi / p_{2}$. The observation plane is placed at a distance $z_{2}$ from the second grating. Because of the symmetry, the problem can be treated as one dimensional. The method employed in the analysis is a series of propagations using the Fresnel approximation from one element of the system to the next. In Appendix A, we analyze the intensity distribution $I_{M}(x)$ at the observation plane for a monochromatic and finite size light source. This analysis has been obtained previously in [13] but, in Appendix A, we have reordered the terms in the quadruple sum so that the pseudoimage formation can be simplified for the case of isolated pseudoimages. Let us assume that the light source is polychromatic with a spectral distribution $g(\lambda)$. The intensity distribution at the observation plane is obtained integrating the intensity produced by each wavelength, Eq. (A1), and weighting it according to the spectrum:

$$
I_{P}(x)=\int I_{M}(x) g(\lambda) \mathrm{d} \lambda .
$$

To obtain analytical solutions, we have assumed a Gaussian spectral distribution,

$$
g(\lambda)=\frac{1}{\sqrt{2 \pi} \Delta \lambda} \exp \left[-\frac{1}{2}\left(\frac{\lambda-\bar{\lambda}}{\Delta \lambda}\right)^{2}\right],
$$

where $\bar{\lambda}$ is the mean wavelength and $\Delta \lambda$ is the spectral width. The integration of Eq. (1) results in

$$
\begin{aligned}
I_{P}(x) \propto & \sum_{N} \sum_{M} \exp \left[i\left(N \alpha_{1}+M \alpha_{2}\right)\right] \operatorname{sinc}\left(N \beta_{1}+M \beta_{2}\right) \\
& \times \sum_{u} \sum_{v} c_{1, u+N / 2} c_{1, u-N / 2}^{*} c_{2, v+M / 2} c_{2, v-M / 2}^{*} \\
& \times \exp \left[-i \pi \Gamma_{N M u v}(\bar{\lambda})\right] \exp \left[-\frac{1}{2}\left(\frac{\Delta \lambda}{\bar{\lambda}}\right)^{2} \Gamma_{N M u v}^{2}(\bar{\lambda})\right],
\end{aligned}
$$

which is valid for all the regimes of pseudoimage formation. The values of $\alpha_{1}, \alpha_{2}, \beta_{1}, \beta_{2}, \Gamma_{N M u v}$ and the 
meaning of the factors are explained in Appendix A. The last factor is not present in Eq. (A1), and it is due to the polychromaticity of the light source.

For the case of isolated pseudoimages, as shown in Appendix A for monochromatic light, the intensity is null for most distances $\left(z_{1}, z_{2}\right)$ except when Eq. (A3) fulfills. The depth of focus of the pseudoimages is given by Eq. (A4). For this regime, the irradiance around a certain pseudoimage $(N, M)$ can be simplified summing only those terms of Eq. (3) that fulfills $Q=-M / N$, resulting in

$$
\begin{aligned}
I_{P}(x) \approx & I_{0,0}+\sum_{k \neq 0} \exp \left[i k\left(N \alpha_{1}+M \alpha_{2}\right)\right] \\
& \times \operatorname{sinc}\left[k\left(N \beta_{1}+M \beta_{2}\right)\right] \\
& \times \sum_{u} \sum_{v} c_{1, u+k N / 2} c_{1, u-k N / 2}^{*} c_{2, v+k M / 2} c_{2, v-k M / 2}^{*} \\
& \times \exp \left[-i \pi \Gamma_{k N, k M, u, v}(\bar{\lambda})\right] \\
& \times \exp \left[-\frac{1}{2}\left(\frac{\Delta \lambda}{\bar{\lambda}}\right)^{2} \Gamma_{k N, k M, u, v}^{2}(\bar{\lambda})\right],
\end{aligned}
$$

where $I_{0,0}=\sum_{n}\left|c_{1, n}\right|^{2} \sum_{m}\left|c_{2, m}\right|^{2}$. Here, the sum $k$ is related to the harmonics of the pseudoimage $(N, M)$. The maximum irradiance of the pseudoimages occurs when Eq. (A3) fulfills. Under this condition, Eq. (ㄴ) results in

$$
\begin{aligned}
& I_{P}(x) \approx I_{0,0}+\sum_{k \neq 0} C_{1, k N / 2} e^{i k q_{1}(M R+N) x} \\
& \quad \times \sum_{v} c_{2, v+k M / 2} c_{2, v-k M / 2}^{*} e^{2 \pi i v k G \lambda} e^{-2(k v G \Delta \lambda)^{2}}, \\
& \text { where } G=\frac{N z_{1}}{p_{1} p_{2}} \frac{N p_{2} z_{0}+M p_{1} z_{1}}{N p_{2} z_{0}+M p_{1}\left(z_{0}+z_{1}\right)}, \\
& C_{1, k N / 2}=\sum_{u=-\infty}^{\infty} c_{1, u+k N / 2} c_{1, u-k N / 2}^{*} .
\end{aligned}
$$

\section{A. Constant-Contrast Pseudoimages with Polychromatic Light}

For polychromatic light most terms of Eq. (5) disappear from a certain threshold distance $\overline{z_{\mathrm{TH}}}$. The reason is that the Gaussian factor vanishes when the distance $z_{1}$ increases. Nevertheless, there exist some terms, which do not disappear since the argument of the Gaussian term is null. That happens when $v=0$. Then $M$ needs to be even, and $v$ runs integer values, $v=\ldots,-1,0,1 \ldots$. When $z_{1} \gg z_{\mathrm{TH}}$ the term $v=0$ is the only that survives. Selecting from Eq. (5) for only those terms with $v=0$ we obtain the stabilized intensity distribution at the exact location of the pseudoimage, which results in

$$
\begin{aligned}
\left.I_{P}(x)\right|_{M \text { even }} \approx & I_{0,0}+\sum_{k \neq 0} C_{1, k N / 2} c_{2, k M / 2} c_{2,-k M / 2}^{*} \\
& \times \exp \left[i k q_{1}(M R+N) x\right] .
\end{aligned}
$$

This equation does not present a dependence on the distance between gratings. In Subsection 2.B we will see how to determine the threshold distance $z_{\mathrm{TH}}$ from which Eq. (6) is valid.

On the other hand, when $M$ is odd, the index $v$ never gets zero in Eq. (5) since $v$ runs semi-integer values. As a consequence, the pseudoimage disappears when $z_{1} \gg z_{\mathrm{TH}}$, and the intensity is $\left.I_{P}(x)\right|_{M \text { odd }} \approx I_{0,0}$. This result explains the behavior numerically predicted in Fig. 8 of [14] for pseudoimages $(1,-2)$ and $(1,-3)$. When polychromatic light is used, pseudoimage $(1,-2)$ presents a high contrast, but pseudoimage $(1,-3)$ disappears.

By means of the simplifications performed in the present paper, we can also determine the contrast of the pseudoimages. When only the first harmonic is considered, the contrast of the stable fringes results in

$$
\text { Contrast }\left.\right|_{M \text { even }}=2 \frac{c_{2, M / 2} c_{2,-M / 2}^{*} \sum_{u} c_{1, u+N / 2} c_{1, u-N / 2}^{*}}{\sum_{n}\left|c_{1, n}\right|^{2} \sum_{m}\left|c_{2, m}\right|^{2}} .
$$

The Fourier coefficients of the gratings are the only factors that determine the contrast of a given pseudoimage, being independent, not only on the distances, but also on properties of the light source, provided that it is polychromatic. As a consequence, the contrast of the pseudoimages depends on the fill factor of the gratings. For example, when two amplitude Ronchi gratings with a $50 \%$ fill factor are used, the predicted contrast for pseudoimage the $(1,-2)$ is $C=0.267$. For the case of a phase grating with a phase shift of $\pi$ between strips and a $50 \%$ fill factor, then the contrast for $(1,-2)$ pseudoimage is $C=0.539$, according to Eq. (7). When this contrast is computed with Eq. (5), it results in $C=0.518$.

\section{B. Threshold Distance}

Equation (6) is valid as long as the terms in Eq. (4) with $v \neq 0$ can be neglected. This happens when the argument of the Gaussian factor is much greater than 1 , that is, $-2(k v G \Delta \lambda)^{2} \gg 1$. This corresponds to a second order inequality in terms of $z_{1}$,

$$
\frac{M}{p_{2}} z_{1}^{2}+\left(\frac{N}{p_{1}} z_{0}-\frac{M p_{1}}{\sqrt{2} k N v \Delta \lambda}\right) z_{1}-\frac{M p_{1}+N p_{2}}{\sqrt{2} k N v \Delta \lambda} z_{0} \gg 0,
$$

which can be easily solved. We call threshold distance $z_{\mathrm{TH}}$ to the distance where the equality holds. The linear series expansion of the solution results in

$$
z_{1} \gg \frac{p_{1} p_{2}}{\sqrt{2} k N v \Delta \lambda}+z_{0} .
$$

As an example, for the case of $z_{0}=0$ we obtain that the threshold distance for each term results in

$$
z_{\mathrm{TH}}=\frac{p_{1} p_{2}}{\sqrt{2} N v_{n v} \Delta \lambda},
$$


where $v_{n v}$ is the first term in Eq. (5) that it is not null, that is, $c_{2, v+k M / 2} c_{2, v-k M / 2}^{*} \neq 0$. For a given pseudoimage $(N, M)$, the threshold distance depends on the characteristics of the second grating. For example, when the grating is binary with a fill factor $50 \%$, then the even diffraction orders are null. For pseudoimage $(1,-2)$, then $v_{n v}=3$. On the other hand, when the fill factor is different from $50 \%$, the even orders are not null and then $v_{n v}=2$.

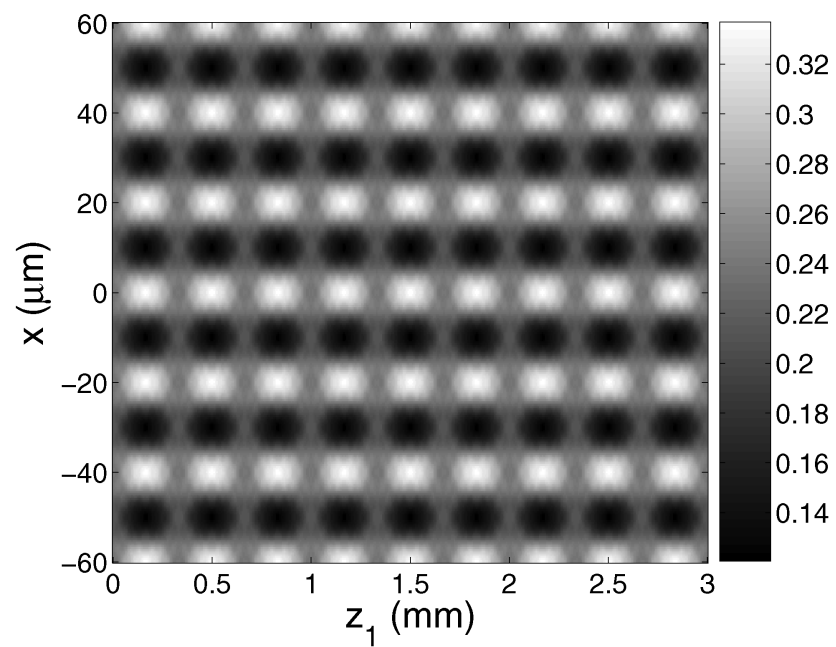

(a)

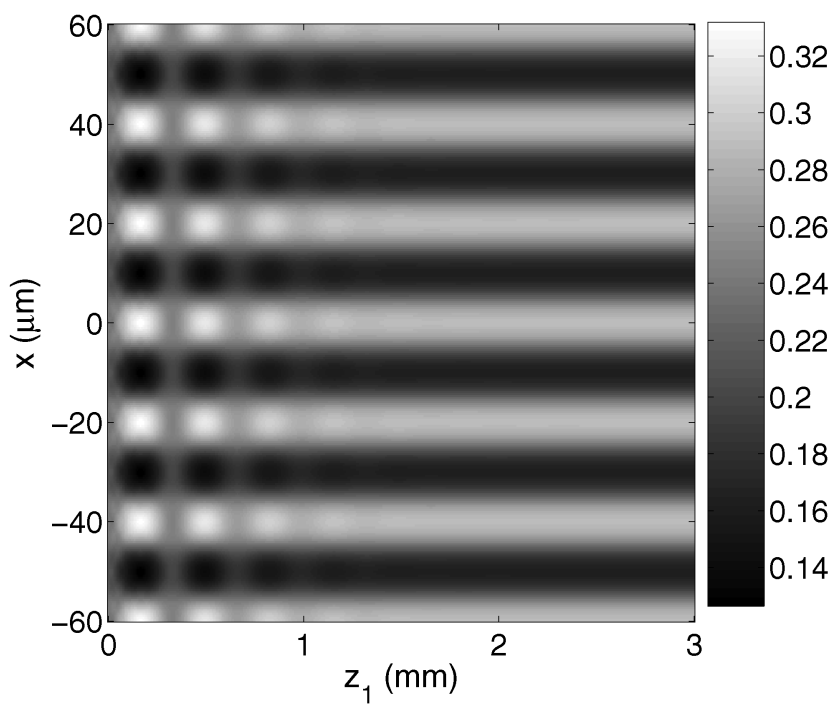

(c)

\section{Numerical Results}

To analyze the effect of the polychromatic light in pseudoimage formation, we have first compared the fringes produced with a monochromatic and a polychromatic light source, using Eq. (3). As an example, the light source presents a mean wavelength $\bar{\lambda}=600 \mathrm{~nm}$ and size $S=300 \mu \mathrm{m}$. Both gratings are amplitude Ronchi gratings and their period is $p_{1}=p_{2}=20 \mu \mathrm{m}$. In Fig. $\underline{2}$, one can see the intensity

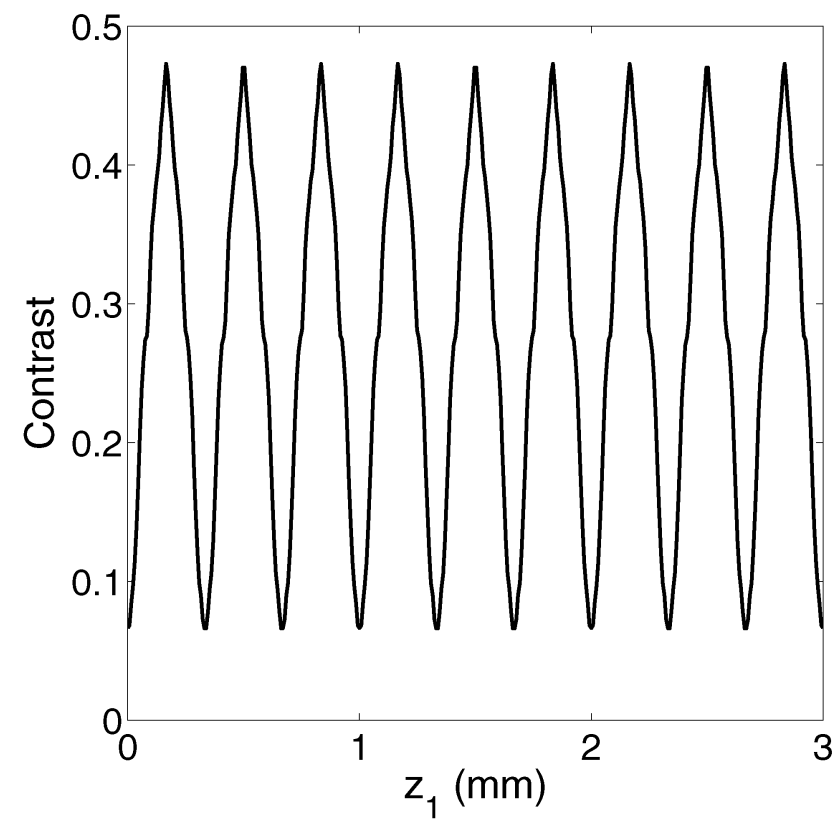

(b)

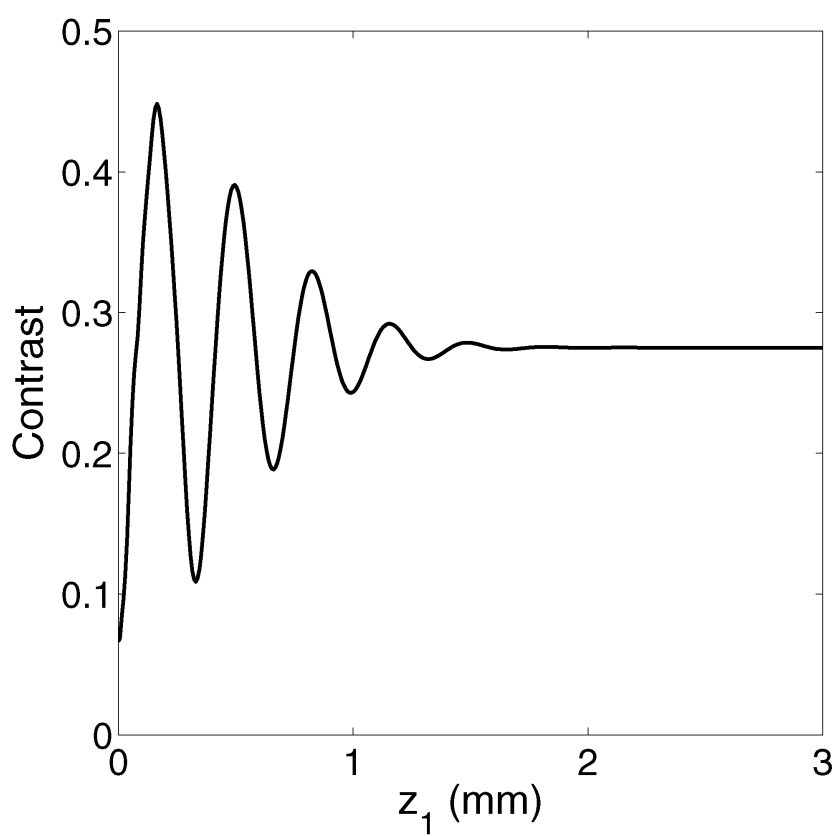

(d)

Fig. 2. Fringes and contrast of pseudoimage $(1,-2)$ for monochromatic (a), (b) and polychromatic (c), (d) light obtained with Eq. (2). The mean wavelength is $\bar{\lambda}=600 \mathrm{~nm}$, the spectral width is $\Delta \lambda=60 \mathrm{~nm}$, the source size is $S=300 \mu \mathrm{m}$, and $z_{0}=0 \mathrm{~mm}$. Both gratings are amplitude Ronchi gratings, $p_{1}=p_{2}=20 \mu \mathrm{m}$. Fringes are obtained for $z_{1}=z_{2}$ (exact location of the pseudoimage). 
profile and the contrast predicted by Eq. (5) for the cases of a monochromatic and a polychromatic light source. The contrast for the monochromatic light fluctuates strongly with the distance between the two gratings. When polychromatic light is used with a spectral width $\Delta \lambda=60 \mathrm{~nm}, \Delta \lambda / \vec{\lambda}=1 / 10$, the threshold distance is approximately $z_{\mathrm{TH}}=1.5 \mathrm{~mm}$ as shown in Fig. 2(d). According to this figure, the stabilized contrast is approximately $C=0.27$, which is in agreement with the result obtained using Eq. (7). The theoretical threshold distance for this case results in $z_{\mathrm{TH}}=1.57 \mathrm{~mm}$, which is also very similar to that obtained in such a figure.

As we have indicated, the threshold distance depends on the fill factor of the grating. In Fig. 3 we can see the contrast of pseudoimage $(1,-2)$ obtained using Eq. (5) for two phase gratings with different fill factors $(50 \%$ and $25 \%)$. As predicted, the threshold distance is different. According to Eq. (10), $z_{\mathrm{TH}}=$ $1.88 \mathrm{~mm}$ for the first case and $z_{\mathrm{TH}}=2 . \overline{82} \mathrm{~mm}$ for the second, which are in good agreement with the results obtained in Fig. $\underline{3}$.

\section{Experimental Results}

To our knowledge, in the literature there is no experimental verification of constant contrast pseudoimages when polychromatic light is used. The experimental setup is equivalent to the scheme

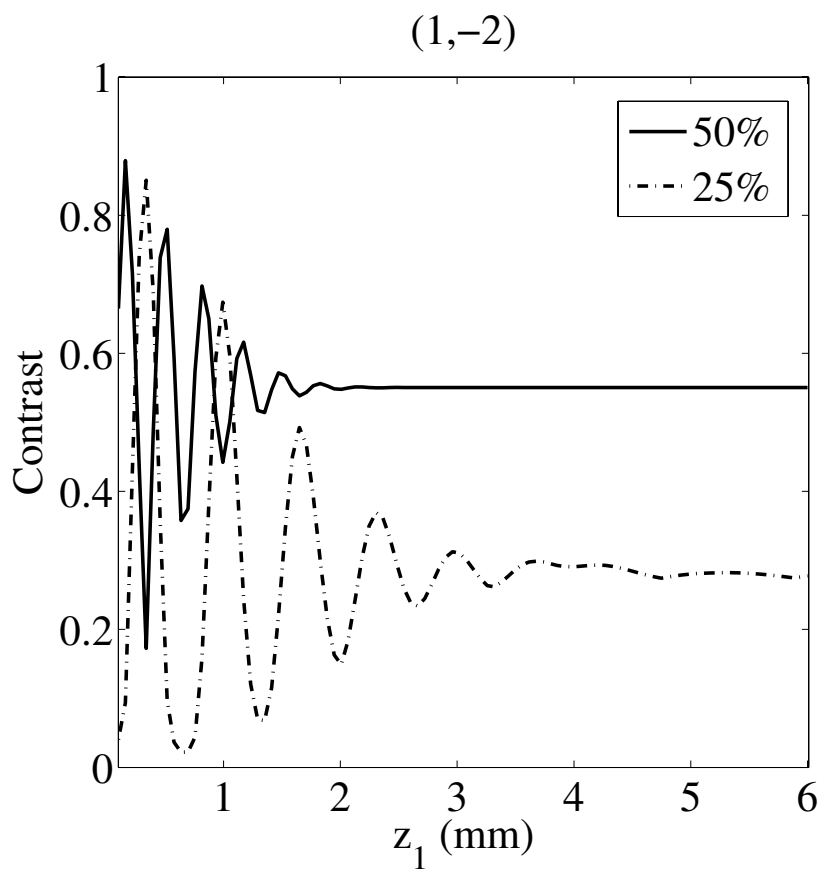

Fig. 3. Contrast of pseudoimage $(1,-2)$ when the first grating G1 is an amplitude grating and the second grating G2 is a phase grating with periods $p_{1}=p_{2}=20 \mu \mathrm{m}$. The mean wavelength is $\bar{\lambda}=600 \mathrm{~nm}$, the spectral width is $\Delta \lambda=50 \mathrm{~nm}$, the source size is $S=300 \mu \mathrm{m}$, and $z_{0}=0 \mathrm{~mm}$. (a) Fill factor of the second grating is 0.5. (b) Fill factor of the second grating is 0.25 . We see that the threshold distance $z_{\mathrm{TH}}$ differs for these two cases as it is theoretically predicted. As shown in the text, the results obtained with Eq. (10) are very similar to those obtained with the simulations.
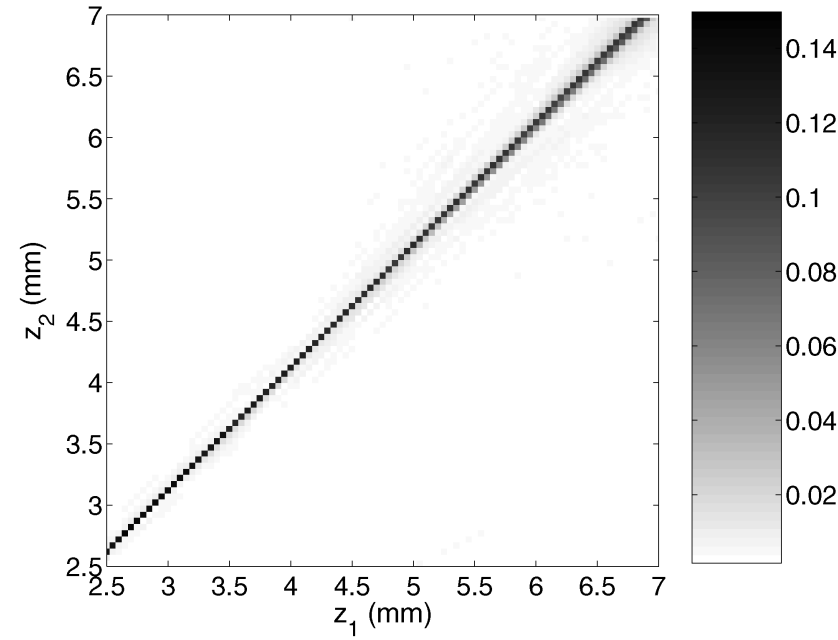

(a)
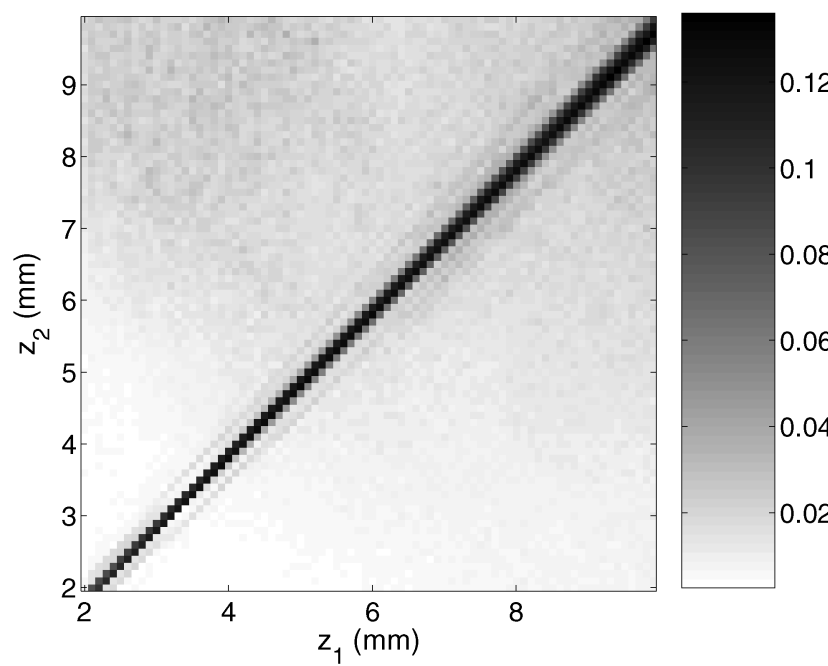

0.1

0.08

0.06

(b)
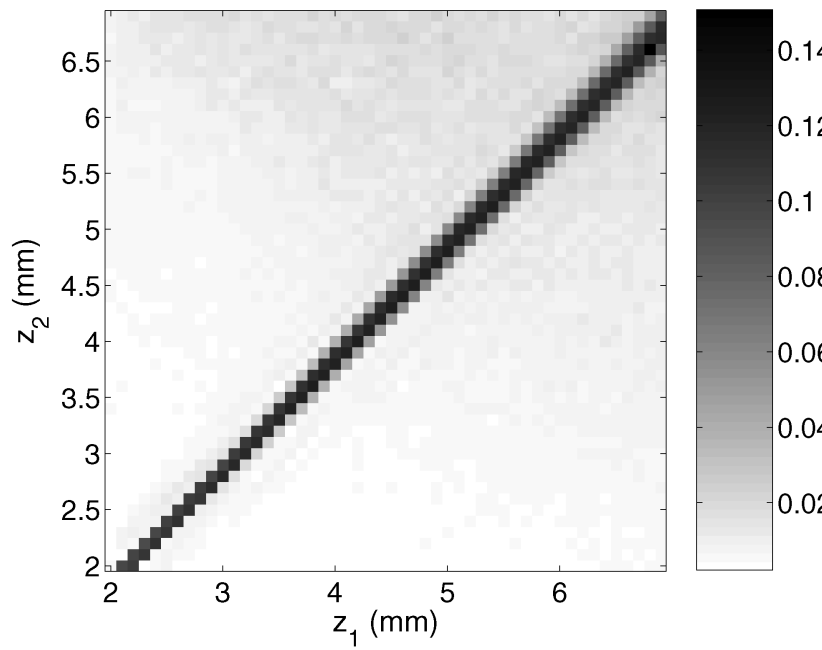

0.14

0.12

(c)

Fig. 4. Experimental contrast of pseudoimage $(1,-2)$ for different LEDs and IREDs (a) HE8811, (b) 600-03V, and (c) HIR333 described in Table 1 . We have used two amplitude Ronchi gratings (fill factor 50\%) with periods $p_{1}=p_{2}=8 \mu \mathrm{m}$. The width of the pseudoimage depends mainly on the LED size. No other pseudoimages have been experimentally found since polychromatic light eliminates them. 
shown in Fig. 4. We have used several LEDs and infrared-emitting diodes (IREDs) with different wavelengths and spectral widths ranging from a white LED $\Delta \lambda / \bar{\lambda} \approx 1 / 4$ to $\Delta \lambda / \bar{\lambda}=1 / 40$ (see Table 1 ).

We have used two identical amplitude Ronchi grâtings of period $p_{1}=p_{2}=8 \mu \mathrm{m}$ made of chromium on a glass. The fill factor of both gratings was $50 \%$. The thickness of the glass was approximately $2 \mathrm{~mm}$. The layer of chromium was not completely opaque, because its transmittance was $T=0.2$. The Fourier coefficients for such gratings were experimentally found resulting in $a_{0}=0.6, a_{1}=0.256$, and $a_{3}=$ -0.0849 . The gratings are displaced with two mobile linear stages, and the fringes are registered in a $\mathrm{CCD}$, a microscope objective $63 \times$ focused to infinite, and a lens with a focus of $45 \mathrm{~mm}$. The distance between the light source and the first grating was $z_{0}=2 \mathrm{~mm}$.

We have measured the contrast for different distances $z_{1}$ and $z_{2}$, which has been computed using a low-pass filter to eliminate high frequency electronic noise. We could not explore the locations $z_{1}, z_{2} \leq$ $2 \mathrm{~mm}$ because of the glass thickness. In Fig. 4, we can see the contrast for several LEDs and IREDs of Table 1 . We have removed the lens that some LEDs present $\mathrm{s}$ o that the location and size of the emitter area are well defined. Only pseudoimage $(1,-2)$ appears, and it does not present contrast fluctuations when a polychromatic light source is used, the pseudoimage is stable with regard to the propagation distance. For this case, the predicted threshold distance is $z_{\mathrm{TH}}=1.5 \mathrm{~mm}$, so the stabilization process of the pseudoimage could not be observed. In fact, other pseudoimages with $M$ have even been experimentally observed, but their contrast is much smaller than for pseudoimage $(1,-2)$, and therefore, it cannot be used in a practical device.

The width of the pseudoimage depends on the source size $S$, in accordance with Eq. (A4). These experimental results are in good agreement with those numerical simulations performed in [14] for the case of the third regime, isolated pseudoimages, where the light source is polychromatic and spatially incoherent.

The experimental contrast for the maximum intensity of the pseudoimage, Eq. (A3), is shown in Fig. 5 for the LEDs and IREDs described in Table 1 . In all the cases, the average experimental stabilized contrast is approximately $C=0.15$, which is in very good agreement with that obtained with Eq. (7), using the

Table 1. LEDs and IREDs Used for Determining the Contrast of $(1,-2)$ Pseudoimages

\begin{tabular}{lccc}
\hline & $\bar{\lambda}$ & $\Delta \lambda$ & $\Delta \lambda / \bar{\lambda}$ \\
& $(\mathrm{nm})$ & $(\mathrm{nm})$ & $\begin{array}{c}\Delta \lambda \\
\text { (Approximately) }\end{array}$ \\
\hline LED 600-03V & 600 & 15 & $1 / 40$ \\
OPE5T85 (Roithner) & 850 & 45 & $1 / 20$ \\
HE8811 (Hitachi) & 820 & 50 & $1 / 15$ \\
HIR333 (Roithner) & 850 & 80 & $1 / 10$ \\
MARL-100059-01 & White & & $1 / 4$ \\
\hline
\end{tabular}

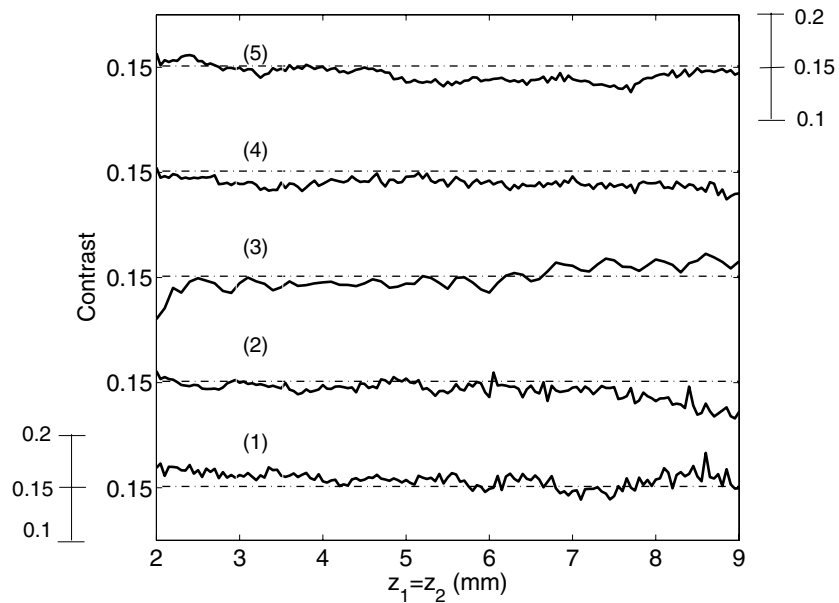

Fig. 5. Experimental contrast of the pseudoimage $(1,-2)$ for the LEDs described in Table 1: (1), LED 600-03V; (2), OPE5T85; (3), HE8811; (4), HIR333; (5), M̄ARL. In all the cases, we have used two Ronchi gratings (fill factor 50\%) with periods $p_{1}=p_{2}=8 \mu \mathrm{m}$. For clarity, the figures have been vertically shifted. Dashed-dotted lines represent the theoretical value (0.1513).

experimental Fourier coefficients of the diffraction grating $C=0.1513$. This shows that the contrast is independent of the light properties and of the distances between components, provided that the light source is polychromatic. For the case of phase gratings the results are equivalent.

\section{Conclusions}

In this paper we have studied the generalized grating imaging configuration when the light source is polychromatic and presents a finite size, such as the case of an LED or an IRED. We have explained why some pseudoimages disappear with the use of polychromatic light and other pseudoimages maintain a high contrast for all distances larger than a threshold value. We have found that pseudoimages $(N, M)$ with $M$ even retain a high contrast when polychromatic light is used. On the other hand, pseudoimages with $M$ odd disappear. We have also obtained simple equations that allow us to determine the contrast of the stabilized pseudoimages and the threshold distance from which the pseudoimage is stable. An experimental analysis has also been performed, which corroborates the theoretical results obtained. These simplified theory and experiments explain the numerical results given in [14], and it can be used to optimize the devices that use generalized grating imaging configuration, making them more tolerant to changes in the gap between gratings.

\section{Appendix A}

The scheme depicted in Fig. 1 is explained at the beginning of Section 2. For the case of a monochromatic light source with a wavelength $\lambda$, a detailed description of the intensity distribution has been obtained by Crespo et al. [13]. We have considered the intensity distribution at the observation plane given in Eq. (17) of such a reference, and we have performed 
the following change of variables: $N=n-n$, $M=m-m \prime, u=n-N / 2$, and $v=m-M / 2$. The reason is that the analysis performed in Section 2 is much simpler with this change of variables. As a consequence, the intensity for monochromatic light can be written as

$$
\begin{aligned}
I_{M}(x)= & \sum_{N} \sum_{M} \exp \left[i\left(N \alpha_{1}+M \alpha_{2}\right)\right] \operatorname{sinc}\left(N \beta_{1}+M \beta_{2}\right) \\
& \times \sum_{u} \sum_{v} c_{1, u+N / 2} c_{1, u-N / 2}^{*} c_{2, v+M / 2} c_{2, v-M / 2}^{*} \\
& \times \exp \left[i \pi \Gamma(\lambda)_{N M u v}\right],
\end{aligned}
$$

where the dummy indices $u$ and $v$ take integer or semi-integer values depending on whether $M$ or $N$ are even or odd, respectively,

$$
\begin{array}{ccc}
R=\frac{q_{2}}{q_{1}}=\frac{p_{1}}{p_{2}}, & \alpha_{1}=q_{1} \frac{z_{0}}{z_{t}} x, & \alpha_{2}=q_{1} R \frac{z_{01}}{z_{t}} x, \\
\beta_{1}=\frac{S}{2} q_{1} \frac{z_{12}}{z_{t}}, & \beta_{2}=\frac{S}{2} q_{1} R \frac{z_{2}}{z_{t}}, & \gamma_{11}=\frac{z_{0} z_{12}}{z_{\lambda} z_{t}}, \\
\gamma_{22}=\frac{z_{2} z_{01}}{z_{\lambda} z_{t}} R^{2}, & \gamma_{12}=\frac{z_{0} z_{2}}{z_{\lambda} z_{t}} R, & z_{\lambda}=\frac{p_{1}^{2}}{\lambda}, \\
z_{t}=z_{0}+z_{1}+z_{2}, & z_{01}=z_{0}+z_{1}, & z_{12}=z_{1}+z_{2},
\end{array}
$$

and $\Gamma(\lambda)_{N M u v}=2 u\left(N \gamma_{11}+M \gamma_{12}\right)+2 v\left(M \gamma_{22}+N \gamma_{12}\right)$. We have explicitly considered the dependence on the wavelength, which is linear. The first exponential is related to the period of the fringes, the sinc function is related to the location and width of pseudoimages, and the second exponential is related to diffraction effects.

When the light source is spatially partially incoherent we are in the third regime [14]. In this case, pseudoimages are isolated, that is, they only present a significant intensity at narrow bands in the $\left(z_{1}, z_{2}\right)$ plane. With Eq. (A1) it is quite simple to determine which terms of the sum belong to a given pseudoimage $(N, M)$. The irradiance around a certain pseudoimage $(N, M)$ can be simplified summing only those terms of Eq. (3) that fulfills $Q=-M / N$, resulting in

$$
\begin{aligned}
I_{P}(x) \approx & I_{0,0}+\sum_{k \neq 0} e^{i k\left(N \alpha_{1}+M \alpha_{2}\right)} \operatorname{sinc}\left[k\left(N \beta_{1}+M \beta_{2}\right)\right] \\
& \times \sum_{u} \sum_{v} c{ }_{1, u+k N / 2} c_{1, u-k N / 2}^{*} c_{2, v+k M / 2} c_{2, v-k M / 2}^{*} \\
& \times e^{-i \pi k \Gamma_{N M u v}(\lambda)},
\end{aligned}
$$

where $I_{0,0}=\sum_{n}\left|c_{1, n}\right|^{2} \sum_{m}\left|c_{2, m}\right|^{2}$ and $k$ runs for the harmonics of the pseudoimage.

With this equation we can determine all the characteristics of a given pseudoimage such as the period, contrast, etc. In particular, we are interested in the location of the pseudoimage $(N, M)$, which is ruled by the sinc term. The maximum intensity of pseudoimage $(N, M)$ is obtained when
$\operatorname{sinc}\left(N \beta_{1}+M \beta_{2}\right)=1$, that is, when $N \beta_{1}+M \beta_{2}=0$. This condition, analyzed in Eq. (19) of [13], results in

$$
z_{2}=\frac{1}{R Q-1} z_{1}
$$

where $Q=-M / N$. That means that pseudoimages are placed in straight lines. The slope of each pseudoimage $(N, M)$ in the $\left(z_{1}, z_{2}\right)$ plane is controlled by the ratio $R=p_{1} / p_{2}$. For each value of $Q$ we obtain different pseudoimages. The harmonics of the pseudoimage $(N, M)$ are $(k N, k M)$ with $k=$ $\ldots,-2,-1,1,2, \ldots$, and they present the same value of $Q$. As a consequence, they are also placed at the same locations.

The width of the pseudoimage $(N, M)$ can be defined as the distance in the $z_{2}$ axis between the two first zeros of the sinc function, that is, $\operatorname{sinc}\left(N \beta_{1}+M \beta_{2}\right)=0$. Normally, the source size is much greater than the period of the gratings, $S \gg p_{1}$. In this case, the depth of focus of the pseudoimage results in

$$
\Delta z_{2} \approx \frac{2 p_{1}}{S} \frac{z_{0}(R M+N)+z_{1} R M}{(R M+N)^{2}} .
$$

For practical distances (millimeters-centimeters), pseudoimages are quite narrow, and they do not overlap. This corresponds to the third regime of [14] where pseudoimages are isolated. In fact, we can always find pairs of integers $(N \prime, M \prime)$ with arbitrarily close $Q$ values that overlap with a given pseudoimage $(N, M)$. Nevertheless, for binary gratings only pseudoimages with small values of $N$ and $M$ present a significant contrast. The reason is that the Fourier coefficients of binary gratings $c_{i, n}$ decrease as $1 / n$. Then, the multiplicative factor in Eq. (3), $c_{1, u+N / 2} c_{1, u-N / 2}^{*} c_{2, v+M / 2} c_{2, v-M / 2}^{*}$ decreases as $1 / u^{2} v^{2}$ and thus the intensity of the pseudoimages.

This work has been supported by the "Codificación óptica de la posición a escala nanométrica: Nuevas tecnologías y dispositivos ópticos" project (DPI200502860) of the Ministerio de Educación y Ciencia of Spain and the "Tecnologías en ecología, alta precisión y productividad, multifuncionalidad, y tecnologías de la información y comunicaciones en Máquina Herramienta" CENIT project of the Ministerio de Industria, Turismo y Comercio. During the realization of this work, L. M. Sanchez-Brea was contracted by the Universidad Complutense de Madrid under the "Ramón y Cajal" research program of the Ministerio de Educación y Ciencia of Spain.

\section{References}

1. L. Liu, "Partially coherent diffraction effect between Lau and Talbot effects," J. Opt. Soc. Am. A 5, 1709-1716 (1988).

2. K. V. Avudainayagam and S. Chitralekha, "Lau effect and beam collimation,” J. Mod. Opt. 44, 175-178 (1997). 
3. M. Tebaldi, L. Angel Toro, and N. Bolognini, "Interferometry based on Lau effect with a grating registered in a photorefractive crystal," Opt. Laser Technol. 31, 127-134 (1999).

4. D. Crespo, J. Alonso, T. Morlanes, and E. Bernabéu, "Optical encoder based on the Lau effect," Opt. Eng. 39, 817-824 (2000).

5. J. Jahns and A. W. Lohmann, "The Lau effect (a diffraction experiment with incoherent illumination)," Opt. Commun. 28, 263-267 (1979).

6. K. Patorski, "The self-imaging phenomenon and its applications," in Progress in Optics, Vol. 27, E. Wolf, ed. (North Holland, 1989), pp. 3-108.

7. S. C. Som and A. Satpathi, "The generalized Lau effect," J. Mod. Opt. 37, 1215-1226 (1990).

8. G. J. Swanson and E. N. Leith, "Analysis of the Lau effect and generalized grating imaging," J. Opt. Soc. Am. A 2, 789-793 (1985).

9. A. Olszak and L. Wronkowski, "Analysis of the Fresnel field of a double diffraction system in the case of two amplitude diffraction gratings under partially coherent illumination," Opt. Eng. 36, 2149-2157 (1997).

10. J. Tu and L. Zhan, "Analysis of general double periodic structure diffraction phenomena based on the ambiguity function," J. Opt. Soc. Am. A 9, 983-995 (1992).

11. D. Crespo, J. Alonso, and E. Bernabeu, "Experimental measurements of generalized grating images," Appl. Opt. 41, 1223-1228 (2002).
12. S. Teng, L. Liu, J. Zu, Z. Luan, and D. Liu, "Uniform theory of the Talbot effect with partially coherent light illumination," J. Opt. Soc. Am. A 20, 1747-1754 (2003).

13. D. Crespo, J. Alonso, and E. Bernabeu, "Generalized grating imaging using an extended monochromatic light source," J. Opt. Soc. Am. A 17, 1231-1240 (2000).

14. L. Garcia-Rodriguez, J. Alonso, and E. Bernabeu, "Grating pseudo-imaging with polychromatic and finite extension sources" Opt. Express 12, 2529-2541 (2004).

15. N. Guérineau, B. Harchaoui, and J. Primot, "Talbot experiment re-examined: demonstration of an achromatic and continuous self-imaging regime," Opt. Commun. 180, 199-203 (2000).

16. N. Guèrineau, E. di Mambro, J. Primot, and F. Alves, "Talbot experiment re-examined: study of the chromatic regime and application to spectrometry," Opt. Express 11, 3310-3319 (2003).

17. L. M. Sanchez-Brea, J. Alonso, and E. Bernabeu, "Continuous pseudoimages for sinusoidal grating imaging using an extended light source," Opt. Commun. 236, 53-58 (2004).

18. L. M. Sanchez-Brea, J. Alonso, J. B. Saez-Landete, and E. Bernabeu, "Analytical model of a double grating system with partial temporal and spatial coherence," in Nano- and MicroMetrology, H. Ottevaere, P. DeWolf, and D. S. Wiersma, eds., Proc. SPIE 5858, 304-311 (2005). 